\title{
Spin-Orbit Interaction and Isotropic Electronic Transport in Graphene
}

\author{
Mahmoud M. Asmar ${ }^{*}$ and Sergio E. Ulloa ${ }^{\dagger}$ \\ Department of Physics and Astronomy and Nanoscale and Quantum Phenomena Institute, Ohio University, Athens, Ohio 45701-2979, \\ USA and Dahlem Center for Complex Quantum Systems and Fachbereich Physik, Freie Universität Berlin, 14195 Berlin, Germany
}

(Received 28 August 2013; published 1 April 2014)

\begin{abstract}
Broken symmetries in graphene affect the massless nature of its charge carriers. We present an analysis of scattering by defects in graphene in the presence of spin-orbit interactions (SOIs). A characteristic constant ratio $(\simeq 2)$ of the transport to elastic times for massless electrons signals the anisotropy of the scattering. We show that SOIs lead to a drastic decrease of this ratio, especially at low carrier concentrations, while the scattering becomes increasingly isotropic. As the strength of the SOI determines the energy (carrier concentration) where this drop is more evident, this effect could help evaluate these interactions through transport measurements in graphene systems with enhanced spin-orbit coupling.
\end{abstract}

DOI: 10.1103/PhysRevLett.112.136602

PACS numbers: 72.80.Vp, 03.65.Pm, 72.10.Fk, 75.76.+j

The discovery of graphene has stimulated numerous theoretical and experimental works [1], opening new doors for promising new technology due to its low dimensionality and high carrier mobility. The low energy electron dynamics is described by two inequivalent points at the Brillouin zone $\left(K\right.$ and $\left.K^{\prime}\right)$ known as Dirac points, since the linear dispersion is equivalent to two-dimensional massless Dirac fermions $[2,3]$.

The importance of graphene on transport devices also motivates the identification and understanding of spin dynamics [4], as an important element in the development of spintronics. In graphene, interface or bulk broken symmetries allow for the existence of two kinds of spinorbit interaction (SOIs) that affect spin dynamics in different ways [5]. The hexagonal arrangement of carbon atoms allows an intrinsic SOI that respects lattice symmetries and can be seen to arise from the atomic SO coupling. This generates a gap in the spectrum, a mass term in the Dirac equation with sign depending on the spin, pseudospin, and Dirac valley [6,7]. An inversion asymmetry in graphene could also generate an extrinsic Rashba SOI, resulting from the effect of substrates, impurities generating $s p^{3}$ distortions—-such as hydrogen, fluorine, or gold-perpendicular electric fields, or lattice corrugations [8-13]. Intercalation of gold under graphene deposited on nickel substrates results in very large Rashba interactions [13], while a large enhancement was observed in weakly hydrogenated samples [14]. In addition, recent theoretical studies have shown that decoration of graphene with heavy atoms such as indium and thallium will result in the enhancement of an intrinsiclike SOI in graphene and the associated quantum spin Hall state [15].

Adsorbed impurities [16,17], as well as lattice vacancies and other local defects in the lattice [18] provide natural short-range scattering centers known as resonant scatterers. Sources of resonant scatterers are also organic groups [19], clusters of impurities [20], or even artificially controlled metallic islands deposited on the surface of graphene [21]. Extensive work has identified the existence of resonant scatterers as the main mechanism limiting carrier mobility in graphene samples [19,22,23]. These conclusions are supported by the insensitivity to screening effects provided by the different substrates used [24,25], by the independence of the ratio of the transport to elastic times to the carrier concentration [26], and by the universal presence of the Raman $D$ peak in graphene devices and its stability after high-temperature annealing of samples [27,28]. Experiments performed by Monteverde et al. [26] used the transport $\left(\tau_{\text {tr }}\right)$ and elastic $\left(\tau_{e}\right)$ scattering times extracted from magnetotransport measurements to probe the nature of the impurities in single and bilayer graphene. The ratio of these two characteristic times, $\xi=\tau_{\text {tr }} / \tau_{e}$, describes at low Fermi energies (low carrier concentration) the degree of angular anisotropy of the scattering process, offering an interesting insight on the type of impurities present in samples. One should comment that other work argues that carrier mobility in graphene is mainly limited by long range scattering from charged impurities [29-32], also related to the formation of electron-hole puddles $[2,24,30,33]$.

Short range scatterers are categorized according to the total cross section $\sigma_{t}$ they produce [26]: "Small cross section scatterers" have $\sigma_{t} \propto k$, where $k$ is the carrier Fermi wave number $\left(k \propto E_{F} \propto \sqrt{n_{c}}\right.$, with $n_{c}$ the carrier density). "Medium cross section scatterers" are referred to in the literature also as resonant scatterers, and display a different dependence, $\sigma_{t} \propto 1 /\left(k \ln ^{2} k\right)$. Finally, the "large total cross section scatterers" or "unitary" are associated with the presence of a long-lived quasibound state [34,35], and exhibit $\sigma_{t} \propto 1 / k$. An important common property shared by all these regimes is that the ratio of the transport to elastic times is determined fully by the conservation of pseudohelicity, leading to a value of 2 at low energies, as we will discuss below. 
We will show that the presence of SOIs leads to an important transformation of scattering processes in graphene, from highly anisotropic (zero backscattering) to more or fully isotropic at low energies, depending on the strength of these interactions. We show that the Rashba SOI results in the appearance of new unitary resonances for short-range scatterers, whenever Rashba coupling is comparable to the Fermi energy. Moreover, we show that the three different types of short range scatterers (off resonant, resonant, and unitary), lead to processes with different levels of angular isotropy, unlike the case with no Rashba SOI when all short range scatterers display similar anisotropy. These findings suggest that transport experiments performed at low carrier concentration could unveil the local enhancement of the Rashba interaction produced by impurities, lattice corrugations, or substrate effects, and provide a direct measurement of its strength.

We consider the presence of intrinsic SOI, affecting the carriers throughout the graphene system, while an extrinsic scatterer generates a local potential obstacle and the corresponding Rashba SOI; the Hamiltonian for this system close to the Dirac points is then given by

$$
H=H_{o}+H_{V}+H_{\mathrm{SO}}+H_{R},
$$

where $H_{o}=\hbar v_{F}\left(\tau_{z} \sigma_{x} p_{x}+\sigma_{y} p_{y}\right)$ describes Dirac fermions in graphene, $H_{\mathrm{SO}}=\Delta_{\mathrm{SO}} \sigma_{z} \tau_{z} s_{z}$ is the intrinsic SOI, $H_{V}=V \Theta(R-r)$ is the scattering potential characterized by strength $V$ over a region $r<R$, and $H_{R}=$ $\lambda_{R}\left(\tau_{z} \sigma_{x} s_{y}-s_{x} \sigma_{y}\right) \Theta(R-r)$ is the Rashba SOI [5] over the same region; here, $\hbar v_{F} \simeq 6.6 \mathrm{eV} \AA$, while $\sigma_{\mu}$ and $s_{\mu}$ are Pauli matrices representing the electron pseudospin $(A, B)$ and spin $(\uparrow, \downarrow)$, respectively, and $\tau_{z}= \pm 1$ identifies the $K$ or $K^{\prime}$ valleys. $\Delta_{\text {SO }}$ and $\lambda_{R}$ are the strengths of intrinsic and Rashba interactions, and $\Theta$ is the Heaviside function. The characteristic size of the scatterers is assumed to be much larger than the lattice spacing in graphene for the continuum Dirac description of graphene to be appropriate, and to neglect intervalley scattering $[2,36]$.

The analytical form of the spinors [37] allows one to use a partial wave decomposition to study the scattering of an incoming flux of electrons along the $x$ direction [38], which takes the asymptotic form away from the scattering center

$$
\psi \approx e^{i k r \cos \theta} \chi_{\mathrm{in}}+\hat{f}(\theta) \frac{e^{i k r}}{\sqrt{r}} \chi_{\mathrm{in}},
$$

where $\chi_{\text {in }}=\left(c_{1}|\uparrow\rangle, c_{2}|\downarrow\rangle\right)^{T}$ is a spinor describing the spin weights of the incoming flux with $\left|\chi_{\text {in }}\right|^{2}=1$, $k=\sqrt{E^{2}-\Delta_{\text {SO }}^{2}} / \hbar v_{F}$, and $\hat{f}(\theta)$ is a matrix containing the different scattering amplitudes. The conservation of total angular momentum $J_{z}=L_{z}+\hbar \tau_{z} \sigma_{z} / 2+\hbar s_{z} / 2$, where $J_{z} \psi_{n}=\hbar n \psi_{n}$ [37], allows consideration of separate partial wave components of the incoming wave with a given spin $s, \psi_{n}^{(-)}|s\rangle$. Hence, the full wave function away from the scattering area is given by

$$
\psi_{n}^{\text {out }}(r, \theta)=\psi_{n}^{(-)}|s\rangle+\sum_{s^{\prime}} S_{n, s s^{\prime}} \psi_{n}^{(+)}\left|s^{\prime}\right\rangle
$$

where $s, s^{\prime}=\uparrow, \downarrow$ and $\psi_{n}^{(+)}$is an outgoing wave. The asymptotic form of the Henkel functions and the JacobyAnger expansion [37] allows one to relate the wave functions in (2) and (3), and characterize the scattered part of the wave function as $\bar{s}=-s$,

$$
\psi_{n}^{\text {sct }}=\frac{e^{-i \pi / 4}}{\sqrt{2 \pi k}}\left(\left(S_{n, s s}-1\right) \psi_{n}^{(+)}|s\rangle+i \bar{s} S_{n, s \bar{s}} \psi_{n}^{(+)}|\bar{s}\rangle\right),
$$

leading to the scattering amplitude matrix

$$
\hat{f}(\theta)=\frac{e^{-i \pi / 4}}{i \sqrt{2 \pi k}} \sum_{n}\left[\begin{array}{ll}
f_{n, \uparrow \uparrow} & f_{n, \downarrow \uparrow} \\
f_{n, \uparrow \downarrow} & f_{n, \downarrow \downarrow}
\end{array}\right] e^{\operatorname{in} \theta},
$$

where $f_{n, s s}=S_{n, s s}-1, f_{n, s \bar{s}}=i \bar{s} S_{n, s \bar{s}}$, and the sum over $n$ in (5) runs over all integers. Conservation of flux for each channel of angular momentum (unitarity of $S$ ), imposes the condition $\left|S_{n, s s}\right|^{2}+\left|S_{n, s \bar{s}}\right|^{2}=1$, so that one can relate the scattering amplitudes to the phase shifts gained during the scattering process by $S_{n, s s} \equiv e^{2 i \delta_{n, s s}} \cos \delta_{n, s \bar{s}}$ and $S_{n, s \bar{s}} \equiv \sin \delta_{n, s \bar{s}}$, where $\delta_{n, s s}$ is the phase for spin preserving processes and $\delta_{n, s \bar{s}}$ is conveniently defined for spin-flipping events [39-41]. The description above, an extension of the partial wave component method [38], allows for the exploration of spin-dependent phenomena [39] and observables such as the differential cross section $\sigma(\theta)$, that explicitly displays the anisotropy of the scattering, the transport cross section $\sigma_{\text {tr }}$, related to the transport mean free time, $\tau_{\mathrm{tr}}^{-1}=n_{\text {imp }} v_{F} \sigma_{\text {tr }}$, and the total cross section $\sigma_{t}$, related to the elastic scattering time, $\tau_{e}^{-1}=n_{\text {imp }} v_{F} \sigma_{t}$, where $n_{\text {imp }}$ is the impurity concentration in the sample. In the presence of SOIs the scattering includes spin-preserving and spinflip events. Correspondingly, all these cross sections are spin-dependent matrices given by

$$
\begin{gathered}
\sigma_{s s^{\prime}}(\theta)=\frac{1}{2 \pi k}\left|\sum_{n} f_{n, s s^{\prime}} e^{\mathrm{in} \theta}\right|^{2}, \\
\sigma_{t, s s^{\prime}}=\frac{1}{k} \sum_{n}\left|f_{n, s s^{\prime}}\right|^{2},
\end{gathered}
$$

and

$$
\sigma_{\mathrm{tr}, s s^{\prime}}=\sigma_{t, s s^{\prime}}-\frac{1}{k} \sum_{n} \operatorname{Re}\left(f_{n, s s^{\prime}} f_{n+1, s s^{\prime}}^{*}\right) .
$$

In the absence of SOIs the pseudohelicity, $\sigma \cdot \mathbf{p} / p$ is a conserved quantity $[2,38]$ and results in the equality $f_{m} \equiv f_{-(m-1)}$, where $m$ is an integer $\left(n=m \mp \frac{1}{2}\right.$ for $\uparrow / \downarrow)[37,38]$, which leads to a vanishing differential cross section at $\theta=\pi$ (Klein tunneling), $\sigma(\theta=\pi)=0$, indicating the anisotropic character of the scattering process and 
the near transparency of barriers in graphene [42-45]. At low carrier concentrations, $k R \ll 1, \quad f_{0} \equiv f_{1}$ and $f_{m \neq 0,1} \approx(k R)^{m}$, leading to $\sigma_{t} \simeq 2 \sigma_{\text {tr }}$, and, therefore, $\xi=\tau_{\text {tr }} / \tau_{e} \simeq 2$. Therefore, scattering of massless Dirac fermions in graphene from short range potential scatterers results in $\xi \simeq 2$, for all $V$ and $R$, as long as the carrier density is small, $k R \ll 1$ [26]. This ratio is fully determined by the number and equal weights of the angular momentum channels contributing to the scattering process. As we will see below, this situation is drastically changed in the presence of SOI.

Graphene with intrinsic SOI.-Graphene systems with uniform intrinsic SOI (for space dependent $\Delta_{\text {SO }}$ see [37]), $\Delta_{\text {SO }} \neq 0$, represent a rich opportunity to explore topological effects. An example of such a system is predicted by appropriate deposition of heavy metal atoms on graphene [15]. In those cases, the eigenstates no longer have a welldefined pseudohelicity, due to the carrier mass generated by the SOI; notice, however, that although this mass is spin dependent, it does not cause intravalley spin-flip processes, and the scattering can still be analyzed in terms of independent spins. The broken pseudo-helicity, however, results in $\delta_{n, s s} \neq \delta_{-(n-1), s s}$. However, effective time reversal symmetry [46] imposes the relations $f_{n, s s}=f_{-n, \bar{s} \bar{s}}$, and $f_{n, s \bar{s}}=f_{-n, \bar{s} s}$, and since spin mixing is not produced by the intrinsic SOI, we have $\delta_{n, s \bar{s}}=\delta_{-n, \bar{s} s}=0$.

As one could suspect, the isotropy of the scattering process depends on the ratio of $\Delta_{\mathrm{SO}} / E$, as shown in Fig. 1: the scattering is anisotropic-with an absence of backscattering-for $\Delta_{\text {SO }}=0$, while it becomes increasingly isotropic with larger $\Delta_{\mathrm{SO}} / E$, and for $\Delta_{\mathrm{SO}} \approx E$, the scattering is equally probable in all directions.

The change in the isotropy of the scattering process is related to the total number of angular momentum channels
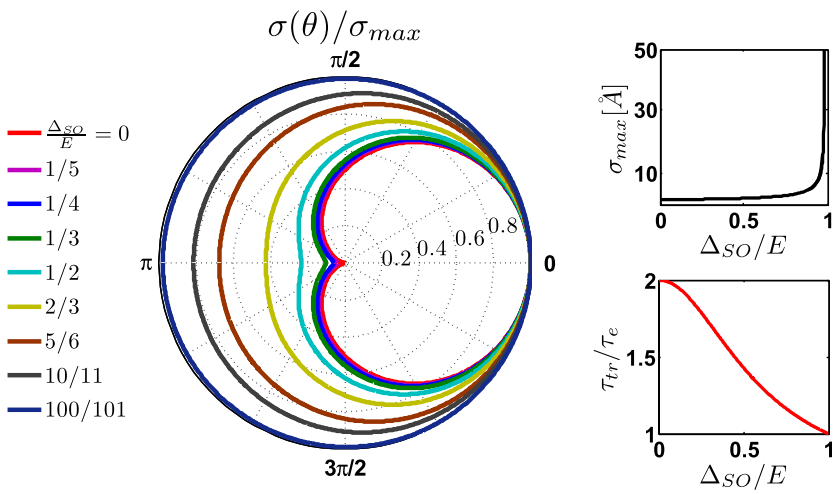

FIG. 1 (color online). Polar plots of the differential cross section, normalized to its maximum, for different values of the intrinsic spin-orbit interaction, $\Delta_{\mathrm{SO}}$-here $E R /\left(\hbar v_{F}\right)=8 \times 10^{-3}$ and $V R /\left(\hbar v_{F}\right)=1.5$. Top inset: $\sigma_{\max }(\theta)$, which increases as $1 /\left[k \ln ^{2}(k R)\right]$ for $\Delta_{\mathrm{SO}} / E \approx 1$, sets the scale used in the polar plots. Bottom inset: Dependence of $\xi=\sigma_{t} / \sigma_{\text {tr }}=\tau_{\text {tr }} / \tau_{e}$ vs $\Delta_{\mathrm{SO}} / E$. Notice that $\sigma(\theta) / \sigma_{\max }$ and $\xi$ do not depend on the value of $V$ in this regime; $V$ only determines the amplitude of $\sigma_{\max }$ in the top inset. contributing to the cross section. For an incoming electron flux with "high" energy, $0 \leq \Delta_{\mathrm{SO}} / E \ll 1$, the system exhibits approximately equal contributions from two scattering channels, $n=0$ and $n=1$ for $\uparrow$ incoming flux (or $n=0$ and $n=-1$ for $\downarrow$ incident flux), and these contributions satisfy $f_{0, \uparrow \uparrow} \approx f_{1, \uparrow \uparrow}$ (or $f_{0, \downarrow \downarrow} \approx f_{-1, \downarrow \downarrow}$ ). In contrast, we observe an increase in the isotropy of the scattering as $E$ decreases, approaching $\Delta_{\text {So }}$, due to the vanishing contribution of the $n=0$ channel to the total cross section, $\vartheta\left(k^{3} R^{4}\right)$, compared to resonant contribution of the $n= \pm 1$ channels $\pi^{2} /\left[k \ln ^{2}(k R)\right]$ [37]. This leads to the "isotropic" ratio of $\xi=\tau_{t r} / \tau_{e} \approx 1$, which is characteristic of the scattering of massive particles at low energies; in other words, one of the spinor components dominates the scattering process in this range of energy and leads to a fully isotropic differential scattering cross section. As $\Delta_{\text {SO }}$ determines the energy scale for which the isotropy would play a larger role, the exploration of decorated graphene samples would be an interesting system in which to test these results [15].

Graphene with Rashba SOI.-We now analyze the case of graphene samples containing scattering centers that also produce Rashba interactions $[9,10,13,14], \Delta_{\text {SO }} \ll \lambda_{R} \neq 0$ [37], allowing spin flip events. This requires a detailed analysis of the spin dependent scattering processes. When $k R \ll 1$, we have two contributing channels, depending on the spin of the incoming particle $(n=0,1$ for spin up, and $n=0,-1$ for spin down), similar to the case discussed above for $\Delta_{\text {SO }} \neq 0$. Effective time reversal symmetry within the Dirac cone allows one to study the scattering of a given spin without loss of generality $[37,46]$.

Curves of total cross section vs scattering potential strength $V$ are shown in Fig. 2(a) for $k R \ll 1$, and different values of the Rashba SOI interaction, $\lambda_{R}$; analytical expressions for the different contributions can be obtained as well [37]. Figure 2(a) shows how the location and number of resonances change in the presence of Rashba SOIs. The resonances at $\chi^{\prime}=\chi_{0} \pm \lambda_{R} R / \hbar v_{F}$ for both $\sigma_{t, s s}$ and $\sigma_{t, s \bar{s}}$, can be identified as resonances of the $n=0$
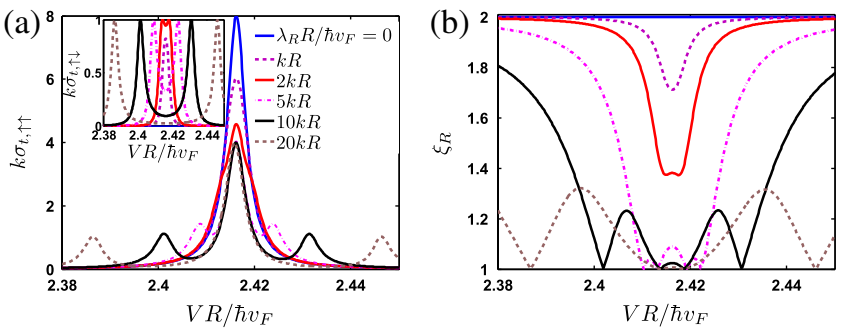

FIG. 2 (color online). (a) Total cross section for spin-preserving processes as a function of the scattering potential shift $V$, for different values of the Rashba SOI, with $k R=1.5 \times 10^{-3}$ and $\Delta_{\mathrm{SO}}=0$ (for $\Delta_{\mathrm{SO}} \neq 0$ see [37]). Inset: Total cross section for spin-flip processes. (b) The ratio $\xi_{R}=\left(\sigma_{t, \uparrow \uparrow}+\sigma_{t, \uparrow \downarrow}\right) /\left(\sigma_{\mathrm{tr}, \uparrow \uparrow}+\right.$ $\left.\sigma_{\mathrm{tr}, \uparrow \downarrow}\right)$ for different values of Rashba SOI [legend as in (a)]. Notice $\xi_{R}=1$ at $\sigma_{t}$ resonances. 
channel, while the resonance at $\chi^{\prime} \approx \chi_{0}+\vartheta\left(\left[\lambda_{R} R /\left(\hbar v_{F}\right)\right]^{2}\right)$ can be identified as coming from the $n=1(-1)$ for $s=\uparrow$ $(\downarrow)$ incoming spin, where $\chi_{0}$ is the location of the unitary resonance in the absence of SOIs. Similarly, Fig. 2(b) demonstrates that the scattering isotropy at resonant values is different from the case of no SOI, by showing that the ratio $\xi_{R}=\left(\sigma_{t, \uparrow \uparrow}+\sigma_{t, \uparrow \downarrow}\right) /\left(\sigma_{\mathrm{tr}, \uparrow \uparrow}+\sigma_{\mathrm{tr}, \uparrow \downarrow}\right)$ takes on different values in the different regimes, being $\xi_{R} \simeq 1$ for unitary resonances, $1<\xi_{R}<2$ for medium scatterers, and $\xi_{R} \simeq 2$ when off-resonance. This qualitative difference arises from the fact that the scattering amplitudes of the two contributing channels are not equal for all the scattering regimes, in contrast to the case of scattering in the absence of the Rashba interaction, where $\xi \simeq 2$ for all regimes, off- and on-resonance.

To further explore the consequences of this SOI-dependent behavior on transport experiments [26], we consider a random distribution of scatterers in a typical graphene sample. The distribution is assumed to be of low density, as we ignore multiple scattering events. Moreover, as the parameter in the theory is $V R$, we assume a random distribution for that quantity in the range 1.5 to 2.4 (in units of $\left.\hbar v_{F}\right)$. For a fixed value $V \simeq 2 \mathrm{eV}$, for example, this would correspond to a variation in $R$ from $\simeq 5$ to $8 \AA$, not unlike those considered before $[9,26]$. The results of such an averaging procedure are shown in Fig. 3, where $\xi_{R}=\left\langle\sigma_{t, \uparrow \uparrow}+\right.$ $\left.\sigma_{t, \uparrow \downarrow}\right\rangle /\left\langle\sigma_{\mathrm{tr}, \uparrow \uparrow}+\sigma_{\mathrm{tr}, \uparrow \downarrow}\right\rangle$ is shown as a function of (Fermi) energy for different values of the Rashba SOI strength $\lambda_{R}$, while $V=2 \mathrm{eV}$ is kept fixed. Notice that the range of $E$ in the figure satisfies $k R<0.24$ for all values shown and can therefore be understood in terms of the analytical expansions above- however, the curves shown are obtained from a full numerical evaluation of the different cross sections that consider multiple channels. As one would expect, as the energy (or carrier density) increases, the ratio $\xi_{R}$ approaches the anisotropic, effectively SOI-free limit, $\approx 2$, while at low energies, $\xi_{R}$ approaches the isotropic scattering limit of 1 . The drop occurs for a characteristic energy given by $\lambda_{R}$, with $\xi_{R} \simeq 1.8$ for $E \simeq \lambda_{R} / 2$; this condition can be traced back to the shifting resonances of the $n=0$ channel under Rashba SOI. One can also analyze the dependence of $\xi_{R}$ on the Rashba coupling for different carrier densities (energies), as shown in the inset of Fig. 3. It is evident that the effect of a small Rashba coupling is more pronounced at lower energies.

From the preceding analysis, it appears that the experimental evaluation of the transport to elastic times ratio at low carrier densities would be able to provide an alternative measure of the effective Rashba SOI present, as produced by impurities and defects, either intrinsic or purposely introduced. Such careful experiments have already explored this ratio [26], and as the carrier density has been reduced down to $E \approx 100 \mathrm{meV}$, it appears the induced Rashba SOI in those samples was well below that number (i.e., $\lambda_{R}<200 \mathrm{meV}$ ), since $\xi_{R} \simeq 2$ over the entire range

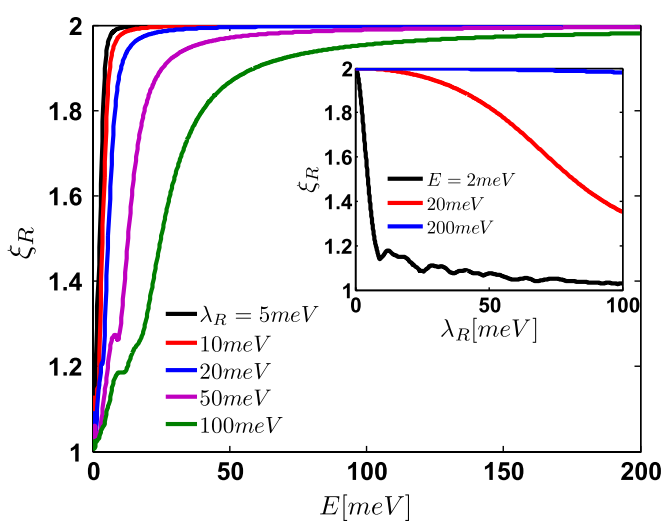

FIG. 3 (color online). The ratio $\xi_{R}$ for 500 randomly sized impurities in the range of $5 \AA \leq R \leq 8 \AA$, for different values of the Rashba coupling and $V=2 \mathrm{eV}$, as a function of carrier energy. Notice a clear drop of $\xi_{R}$ from 2 for $E<\lambda_{R} / 2$. Inset: $\xi_{R}$ as a function of Rashba coupling for different energies.

explored. We believe it would be interesting to repeat those experiments in systems with higher mobility, such as graphene on boron nitride substrates, which may allow reaching even lower carrier densities without large inhomogeneities. Considering that in systems with adatoms the expected SOI is $\lambda_{R} \approx 10 \mathrm{meV}[9,10,14]$, this requires rather low carrier densities, such as those attained on boron nitride substrates $[47,48]$.

We should comment that the observed renormalization of the Fermi velocity near the Dirac point $[47,48]$, which sees the velocity increase as the energy (or carrier density) drops, should result in $\xi_{R}$ dropping down from 2 at a higher energy than in the absence of the velocity renormalization (for a given $\lambda_{R}$, and assuming a large enough $V R$, so that $v_{F}$ rescaling at $V$ is negligible).

In conclusion, we have shown that SOIs in graphene lead to clear signatures in the scattering processes and therefore to observable consequences in electronic transport. The drop in value of the ratio of transport to elastic times from its known value of $\simeq 2$ reflects the presence of SOI, with the ratio dropping to $\simeq 1$ as $E_{F}$ falls close to the SOI energy scale. We have also shown qualitative changes in the number and nature of resonances produced in scattering due to impurities and the Rashba SOI they induce. Three different regimes of scattering can be distinguished based on the levels of isotropy they produce, with the isotropy becoming more pronounced at low carrier concentrations. Measuring the ratio of scattering times with precision at low carrier densities should enable the experimental characterization of impurity-induced spin-orbit interactions.

We thank N. Sandler and M. Zarea for helpful discussions. This work was supported in part by NSF PIRE, and NSF CIAM/MWN Grant No. DMR-1108285. We are grateful for the welcoming environment at the Dahlem Center and the support of the A. von Humboldt Foundation. 
asmar@phy.ohiou.edu †ulloa@ohio.edu

[1] K. S. Novoselov, A. K. Geim, S. V. Morozov, D. Jiang, Y. Zhang, S. V. Dubonos, I. V. Grigorieva, and A. A. Firsov, Science 306, 666 (2004).

[2] A. H. Castro Neto, F. Guinea, N. M. R. Peres, K. S. Novoselov, and A. K. Geim, Rev. Mod. Phys. 81, 109 (2009).

[3] G. W. Semenoff, Phys. Rev. Lett. 53, 2449 (1984).

[4] M. H. D. Guimares, A. Veligura, P. J. Zomer, T. Maassen I. J. Vera-Marun, N. Tombros, and B. J. van Wees, Nano Lett. 12, 3512 (2012).

[5] C. L. Kane and E. J. Mele, Phys. Rev. Lett. 95, 226801 (2005).

[6] H. Min, J. E. Hill, N. A. Sinitsyn, B. R. Sahu, L. Kleinman, and A. H. MacDonald, Phys. Rev. B 74, 165310 (2006).

[7] S. Konschuh, M. Gmitra, and J. Fabian, Phys. Rev. B 82, 245412 (2010).

[8] N. Tombros, C. Jozsa, M. Popinciuc, H. T. Jonkman, and B. J. van Wees, Nature (London) 448, 571 (2007).

[9] A. H. Castro Neto and F. Guinea, Phys. Rev. Lett. 103, 026804 (2009).

[10] K. Pi, W. Han, K. M. McCreary, A. G. Swartz, Y. Li, and R. K. Kawakami, Phys. Rev. Lett. 104, 187201 (2010).

[11] D. Huertas-Hernando, F. Guinea, and A. Brataas, Phys. Rev. B 74, 155426 (2006).

[12] J.-S. Jeong, J. Shin, and H.-W. Lee, Phys. Rev. B 84, 195457 (2011).

[13] D. Marchenko, A. Varykhalov, M. Scholz, G. Bihlmayer, E. Rashba, A. Rybkin, A. Shikin, and O. Rader, Nat. Commun. 3, 1232 (2012).

[14] J. Balakrishnan, G. Kok Wai Koon, M. Jaiswal, A. H. Castro Neto, and B. Ozyilmaz, Nat. Phys. 9, 284 (2013).

[15] C. Weeks, J. Hu, J. Alicea, M. Franz, and R. Wu, Phys. Rev. X 1, 021001 (2011).

[16] J. P. Robinson, H. Schomerus, L. Oroszlány, and V. I. Fal'ko, Phys. Rev. Lett. 101, 196803 (2008).

[17] D. C. Elias, R. R. Nair, T. M. G. Mohiuddin, S. V. Morozov, P. Blake, M. P. Halsall, A. C. Ferrari, D. W. Boukhvalov, M. I. Katsnelson, A. K. Geim, and K. S. Novoselov, Science 323, 610 (2009).

[18] J.-H. Chen, W. G. Cullen, C. Jang, M. S. Fuhrer, and E. D. Williams, Phys. Rev. Lett. 102, 236805 (2009).

[19] T. O. Wehling, S. Yuan, A. I. Lichtenstein, A. K. Geim, and M. I. Katsnelson, Phys. Rev. Lett. 105, 056802 (2010).

[20] M. I. Katsnelson, F. Guinea, and A. K. Geim, Phys. Rev. B 79, 195426 (2009).

[21] B. M. Kessler, Ç. Ö. Girit, A. Zettl, and V. Bouchiat, Phys. Rev. Lett. 104, 047001 (2010).

[22] M. Titov, P. M. Ostrovsky, I. V. Gornyi, A. Schuessler, and A. D. Mirlin, Phys. Rev. Lett. 104, 076802 (2010).

[23] A. Ferreira, J. Viana-Gomes, J. Nilsson, E. R. Mucciolo, N. M. R. Peres, and A. H. Castro Neto, Phys. Rev. B 83, 165402 (2011).

[24] L. A. Ponomarenko, R. Yang, T. M. Mohiuddin, M. I. Katsnelson, K. S. Novoselov, S. V. Morozov, A. A. Zhukov, F. Schedin, E. W. Hill, and A. K. Geim, Phys. Rev. Lett. 102, 206603 (2009).
[25] N. J. G. Couto, B. Sacépé, and A. F. Morpurgo, Phys. Rev. Lett. 107, 225501 (2011).

[26] M. Monteverde, C. Ojeda-Aristizabal, R. Weil, K. Bennaceur, M. Ferrier, S. Guéron, C. Glattli, H. Bouchiat, J. N. Fuchs, and D. L. Maslov, Phys. Rev. Lett. 104, 126801 (2010).

[27] Z. H. Ni, L. A. Ponomarenko, R. R. Nair, R. Yang, S. Anissimova, I. V. Grigorieva, F. Schedin, P. Blake, Z. X. Shen, E. H. Hill, K. S. Novoselov, and A. K. Geim, Nano Lett. 10, 3868 (2010).

[28] A. Eckmann, A. Felten, A. Mishchenko, L. Britnell, R. Krupke, K. S. Novoselov, and C. Casiraghi, Nano Lett. 12, 3925 (2012).

[29] J.-H. Chen, C. Jang, S. Adam, M. S. Fuhrer, E. D. Williams, and M. Ishigami, Nat. Phys. 4, 377 (2008).

[30] S. Adam, E. H. Hwang, V. M. Galitski, and S. Das Sarma, Proc. Natl. Acad. Sci. U.S.A. 104, 18392 (2007).

[31] C. Jang, S. Adam, J.-H. Chen, E. D. Williams, S. Das Sarma, and M. S. Fuhrer, Phys. Rev. Lett. 101, 146805 (2008).

[32] F. Chen, J. Xia, and N. Tao, Nano Lett. 9, 1621 (2009).

[33] J. Martin, N. Akerman, G. Ulbricht, T. Lohmann, J. H. Smet, K. von Klitzing, and A. Yacoby, Nat. Phys. 4, 144 (2008).

[34] V. M. Pereira, F. Guinea, J. M. B. Lopes dos Santos, N. M. R. Peres, and A. H. Castro Neto, Phys. Rev. Lett. 96, 036801 (2006).

[35] A. Matulis and F. M. Peeters, Phys. Rev. B 77, 115423 (2008).

[36] T. Ando, T. Nakanishi, and R. Saito, J. Phys. Soc. Jpn. 67, 2857 (1998).

[37] See Supplemental Material at http://link.aps.org/ supplemental/10.1103/PhysRevLett.112.136602 for the analytical form of the eigenstates, analysis of the symmetries, and different scattering configurations.

[38] D. S. Novikov, Phys. Rev. B 76, 245435 (2007).

[39] M. M. Asmar and S. E. Ulloa, Phys. Rev. B 87, 075420 (2013).

[40] D. Huertas-Hernando, F. Guinea, and A. Brataas, Phys. Rev. Lett. 103, 146801 (2009).

[41] H. Ochoa, A. H. Castro Neto, and F. Guinea, Phys. Rev. Lett. 108, 206808 (2012).

[42] S. E. Ulloa and G. Kirczenow, Phys. Rev. Lett. 56, 2537 (1986).

[43] M. I. Katsnelson, K. S. Novoselov, and A. K. Geim, Nat. Phys. 2, 620 (2006).

[44] V. V. Cheianov, V. Fal'ko, and B. L. Altshuler, Science 315, 1252 (2007).

[45] A. F. Young and P. Kim, Nat. Phys. 5, 222 (2009).

[46] C. W. J. Beenakker, Rev. Mod. Phys. 80, 1337 (2008).

[47] C. R. Dean, A. F. Young, I. Meric, C. Lee, L. Wang, S. Sorgenfrei, K. Watanabe, T. Taniguchi, P. Kim, K. L. Shepard, and J. Hone, Nat. Nanotechnol. 5, 722 (2010).

[48] D. C. Elias, R. V. Gorbachev, A. S. Mayorov, S. V. Morozov, A. A. Zhukov, P. Blake, L. A. Ponomarenko, I. V. Grigorieva, K. S. Novoselov, F. Guinea, and A. K. Geim, Nat. Phys. 7, 701 (2011). 